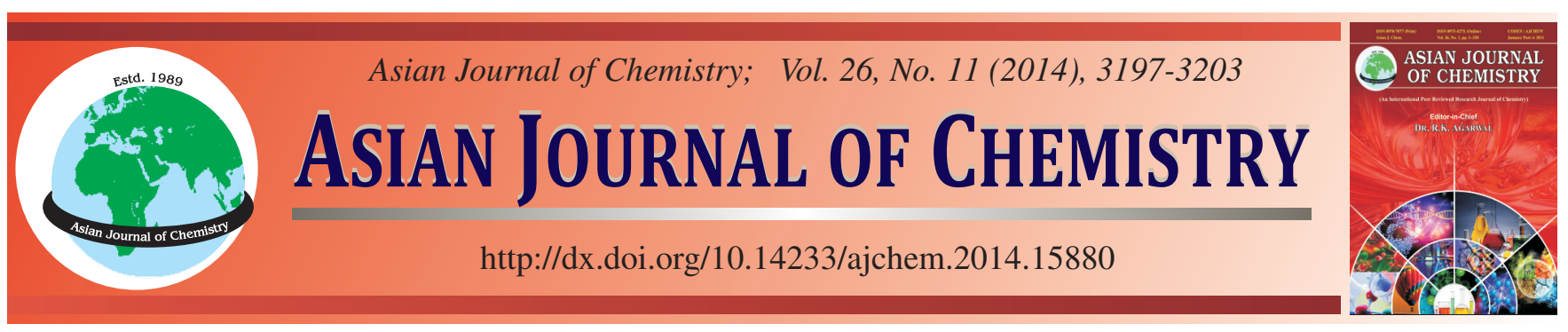

\title{
Synthesis of New 2-(N'-Allylidene-hydrazino)quinazolinones and 2-(4,5-Dihydropyrazolyl)- quinazolinones and Their Antimicrobial and Antifungal Activity Screening
}

\author{
Nezire Saygili ${ }^{1, *}$, Melike Ekizoglu² $^{2}$ and Ceren Erdogdu ${ }^{2}$
}

${ }^{1}$ Department of Basic Pharmaceutical Sciences, Faculty of Pharmacy, Hacettepe University, Sihhiye, 06100 Ankara, Turkey ${ }^{2}$ Department of Pharmaceutical Microbiology, Faculty of Pharmacy, Hacettepe University, Sihhiye, 06100 Ankara, Turkey

*Corresponding author: Fax: +90 312 3114777; Tel: +90 312 3054016-18; E-mail: nezires@ hacettepe.edu.tr

Novel 2-[N'-(1,3-disubstituted-phenyl)allylidene]hydrazine-3-ethyl-3H-quinazolin-4-one (8) and 2-(3,5-disubstituted-phenyl-4,5-dihydropyrazol-1-yl)-3-ethyl-3H-quinazolin-4-one (9) compounds have been prepared and tested for antibacterial and antifungal activities. ${ }^{1} \mathrm{H}$ NMR, ${ }^{13} \mathrm{C}$ NMR, elemental analysis and mass spectroscopy methods have been used to identify molecular structures of the newly synthesized compounds. For screening antibacterial and antifungal activities of the new compounds, minimum inhibitory concentration (MIC) values were evaluated against the Staphylococcus aureus, Escherichia. coli, Pseudomonas aeruginosa, Enterococcus faecalis, Candida albicans,

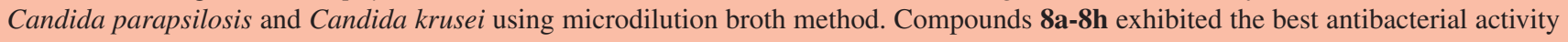
against E. faecalis.

Keywords: Activity, Antifungal, Antimicrobial, Pyrazoline, Quinazolinone, Synthesis.

\section{INTRODUCTION}

The 4- $(3 H)$ quinazolinone compounds are a class of heterocyclic compounds that have wide variety of biological activities ${ }^{1-4}$. The biological importance of these heterocyclics has lead synthetic organic chemists to explore new synthetic routes and thus their syntheses are available in literature ${ }^{5-7}$. A brief search on the pharmaceutical activities of quinazolin-4(3H)-ones showed antiinflammatory $^{8}$, antitumor ${ }^{9}$, anti $\mathrm{HIV}^{10}$, antibacterial ${ }^{11}$, CNS depressant $^{12}$ and anticonvulsant ${ }^{12,13}$ activities. $3 H$-Quinazoline4-one framework is commonly found unit in natural product alkaloids, such as L-vasicinone (1) ${ }^{14}$ and drugs such as diuretic and antihypertensive metolazone $(\mathbf{2})^{15}$. On the other hand, it was found that not only quinazolinones showed diverse biological activities, but also pyrazole moiety possesses some important activities. Pyrazolines are five-membered heterocyclics having two adjacent nitrogen atoms within the ring and display a wide spectrum of pharmacological activities ${ }^{16}$.<smiles>O=c1c2ccccc2nc2n1CC[C@H]2O</smiles><smiles>Cc1ccccc1-n1c(C)nc2cc(Cl)c(S(N)(=O)=O)cc2c1=O</smiles>

Pyrazolyl-4(3H)quinazolinones were also synthesized and screened for some biological activities ${ }^{17,18} \cdot 2$-(1H-pyrazol-1-yl)4-(3H)quinazolinones possess antitubercular, antihistaminic, platelet aggregation inhibitor and microbicidal activities $(\mathbf{3})^{19}$.<smiles>[R][X]c1cccn1-c1nc2ccccc2c(=O)[nH]1</smiles>

In view of the broad spectrum of pharmacological activity of quinazolinone derivatives, we previously published certain substituted quinazolinone derivatives ${ }^{18}$. In this work, we aimed to synthesize some new 2-( $\mathrm{N}^{\prime}$-allylidene-hydrazino)quinazolinone and 2-(4,5-dihydropyrazolyl)quinazolinone compounds and investigate their antimicrobiological activities.

\section{EXPERIMENTAL}

2-Thioxoquinazolinone molecule which was formed from antranilic acid $^{18,20}$, was used as starting material in this chemistry. 2-Thioxoquinazolinones could also be prepared from 2-aminobenzoic acid methyl ester ${ }^{21,22}$ and isatoic anhydride ${ }^{23}$. In our 
<smiles>CCn1c(N)nc2ccccc2c1=O</smiles>

Scheme-I: Synthesis of 3-ethyl-2-hydrazino-3H-quinazolin-4-one (6)

synthetic procedure 3-ethyl-2-thioxo-2,3-dihydro- $1 \mathrm{H}$-quinazolin4-one (5) was synthesized by refluxing anthranilic acid (4) and ethyl isothiocyanate and then $\mathbf{5}$ was transformed to 3-ethyl2-hydrazino-3H-quinazolin-4-one (6) by refluxing with hydrazine hydrate $^{18,24}$ (Scheme-I).

Various $\alpha, \beta$-unsaturated ketones (7) was further reacted with 6 to give hydrazino-3-ethyl-4(3H)quinazolinone (8) derivatives. In the last step 2-[3,5-bis-(substituted-phenyl)-4,5dihydro-pyrazol-1-yl]-3-ethyl-3H-quinazolin-4-one (9) compounds were produced by refluxing hydrazone derivatives in anhydrous acetic acid (Scheme-II).

All reagents were of commercial quality and reagent quality solvents were used without further purification. ${ }^{1} \mathrm{H}$ and ${ }^{13} \mathrm{C}$ NMR spectra were determined on a Bruker DPX $400 \mathrm{MHz}$ FT spectrometer. Mass spectra were obtained on an Agilent 5973 Network Mass Selective Detector via HPP7-M Direct Insertion Probe. IR spectra $(\mathrm{KBr})$ were recorded on a Shimadzu FT-IR DR-8001 FT infrared spectrophotometer. The purity of the compounds was assessed by thin layer chromatography on silica gel 60 F254. Column chromatography was conducted on silica gel 60 (mesh size 0.063-0.200 mm). Melting points were measured on a Thomas Hoover Capillary Melting Point Apparatus in an open capillary.
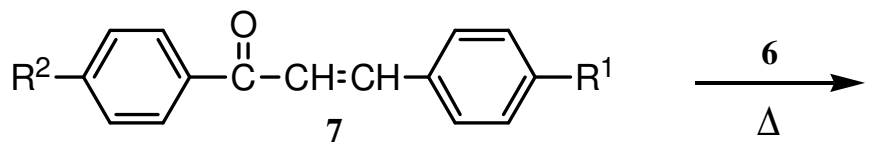
a: $R^{1}=\mathrm{H}, \mathrm{R}^{2}=\mathrm{CH}_{3}$
c: $\mathrm{R}^{1}=\mathrm{CH}_{3}, \mathrm{R}^{2}=\mathrm{CH}_{3}$
e: $R^{1}=H, R^{2}=C l$
$g: \mathrm{R}^{1}=\mathrm{CH}_{3}, \mathrm{R}^{2}=\mathrm{Cl}$

b: $\mathrm{R}^{1}=\mathrm{Cl}, \mathrm{R}^{2}=\mathrm{CH}_{3}$

$\mathrm{d}: \mathrm{R}^{1}=\mathrm{Br}, \mathrm{R}^{2}=\mathrm{CH}_{3}$

$\mathrm{f}: \mathrm{R}^{1}=\mathrm{Cl}, \mathrm{R}^{2}=\mathrm{Cl}$

$\mathrm{h}: \mathrm{R}^{1}=\mathrm{Br}, \mathrm{R}^{2}=\mathrm{C}$
The starting materials, 3-ethyl-2-thioxo-2,3-dihydro- $1 H$ quinazolin-4-one $\mathbf{5}^{25}$, 3-ethyl-2-hydrazino-3H-quinazolin-4one $6^{26,27}$ and $\alpha, \beta$-unsaturated ketone derivatives (chalcones) $7^{28,29}$, were prepared according to the methods given in the literature.

2-[N'-(1,3-diphenyl-allylidene)-hydrazino]-3-ethyl-3Hquinazolin-4-ones (8): A mixture of 7 (1 mmol) and 3-ethyl2-hydrazino-3H-quinazolin-4-one (6) (204 mg, $1 \mathrm{mmol}$ ) and $2 \mathrm{~mL}$ of acetic acid in $n$-propanol $(100 \mathrm{~mL})$ was stirred under reflux for 4-7 h. After the reaction was cooled to room temperature, the resulting residue was filtered, washed with $n$-propanol and dried. Recrystallization of the crude product from ethanol gave $\mathbf{8}$ as a yellow solid.

2-(3,5-Diphenyl-4,5-dihydro-pyrazol-1-yl)-3-ethyl-3Hquinazolin-4-ones (9): A solution of 2-[N'-(1,3-diphenylallylidene)hydrazino]-3-ethyl-3H-quinazolin-4-one (8) $(0.5$ mmol) in glacial acetic acid was stirred under reflux for $72 \mathrm{~h}$. The resulting solution was poured into ice-water, neutralized with concentrated $\mathrm{NaOH}$ solution and extracted with ethyl acetate. The organic phase was dried over magnesium sulphate, filtered and evaporated to dryness to yield the oily residue. The crude product was further purified by chromatography<smiles>[R2]c1ccc(/C=C/C(=N/Nc2nc3ccccc3c(=O)n2CC)c2ccc([R2])cc2)cc1</smiles>

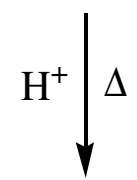<smiles>[R7]c1ccc(C2=NN(c3nc4ccccc4c(=O)n3CCCCCC)C(c3ccc([R])cc3)C2)cc1</smiles> 
on a silica gel column (elution with chloroform) followed by crystallization from diethyl ether to give $\mathbf{9}$ as a white solid.

Antimicrobial activity: Minimum inhibitory concentrations (MICs) were tested by microdilution broth method recommended by Clinical and Laboratory Standards Institute $(\mathrm{CLSI})^{30,31}$. According to these procedure, antibacterial activity was determined against following microorganisms: Staphylococcus aureus (ATCC 29213), Enterococcus faecalis (ATCC 29212), Escherichia coli (ATCC 25922) and Pseudomonas aeruginosa (ATCC 27853). The following yeast-like fungi were used for antifungal activity studies: Candida albicans (ATCC 90028), Candida parapsilosis (ATCC 90018) and C. krusei (ATCC 6258).

The stock solutions of the synthesized compounds were prepared in dimethyl sulfoxide (DMSO). Test was performed in Mueller-Hinton Broth (MHB) (BBL, MD, USA) for bacteria. Fungi were cultivated in Sabouraud Dextrose Agar (SDA) (Merck). RPMI-1640 medium (ICN-Flow, Aurora, OH-USA) with L-glutamin, buffered with 3-(N-morpholino)propane sulphonic acid (MOPS) (Buffer-ICN-Flow, Aurora, OH-USA) at $\mathrm{pH}=7.4$ was used as the test medium. The final inoculum densities were $5 \times 10^{5} \mathrm{cfu} / \mathrm{mL}$ for bacteria and $0.5-2.5 \times 10^{3}$ $\mathrm{cfu} / \mathrm{mL}$ for fungi. The dilutions in the test medium were prepared in the wells of the microtiter plates at the required concentration of 1024-1.0 $\mu \mathrm{g} / \mathrm{mL}$ and for reference compounds at $64-0.0625 \mu \mathrm{g} / \mathrm{mL}$. Fluconazole and ciprofloxacin were used as the reference compounds for fungi and bacteria, respectively. The microtiter plates were incubated at $35^{\circ} \mathrm{C}$ for 18 $24 \mathrm{~h}$ for bacteria and $48 \mathrm{~h}$ for fungi. MICs were defined as the lowest concentration of the compound that inhibited visible growth of the microorganisms. It was established that dilution of DMSO lacked antimicrobial activity against any of the test microorganisms.

\section{RESULTS AND DISCUSSION}

New quinazolinone compounds have been synthesized and their structures were proven by ${ }^{1} \mathrm{H}$ - and ${ }^{13} \mathrm{C}$ NMR, mass spectra, elemental analysis and all spectral data are in accordance with the assigned structures. The first step involves the ring closure reaction to form 2-thioxoquinazoline which in turn converted to 2-hydrazinoquinazoline via nucleophilic addition and then elimination reaction. Amino group of $\mathbf{6}$ gives 1,4-addition reaction with $\alpha, \beta$-unsaturated ketones (7) to afford hydrazone (8) derivatives. Acid catalyzed pyrazole ring formation occurs with subsequent addition of NH group on olefinic bond to give 2-pyrazolylquinazolinones (9). In the ${ }^{1} \mathrm{H}$ NMR spectra of the compounds 5, olefinic protons $\left(2^{\prime}-\mathrm{CH}\right.$ and $\left.3^{\prime}-\mathrm{CH}\right)$ appeared as doublets at about 6.63-6.93 and 7.82-8.10 ppm, respectively $\left(J_{\text {trans }}=16.2-16.8 \mathrm{~Hz}\right)$. After the ring closure, ring protons $\left(4^{\prime}-\mathrm{CH}_{\mathrm{a}}\right.$ and $\left.4^{\prime}-\mathrm{CH}_{\mathrm{b}}\right)$ of the compounds 9 showed double doublets at around 3.59-3.78 $\left(J_{\mathrm{dd}}=11.2-11.4\right.$ and 16.8$17.0 \mathrm{~Hz})$ and $3.16-3.32 \mathrm{ppm}\left(J_{\mathrm{dd}}=12.0\right.$ and $\left.16.8-17.0 \mathrm{~Hz}\right)$ and also 5'-CH gave triplet at about 5.79-5.96 ppm $\left(J_{\mathrm{t}}=11.2-\right.$ $12.0 \mathrm{~Hz}$ ) due to vicinal coupling with the two magnetically nonequivalent protons of the methylene group. The quinazolinone and phenyl protons were observed at the expected chemical shifts and integral values. In the ${ }^{13} \mathrm{C}$ NMR spectra of the compound 8c, olefinic carbons $\left(2^{\prime}-\mathrm{C}\right.$ and $\left.3^{\prime}-\mathrm{C}\right)$ appeared at 138.69 and $119.84 \mathrm{ppm}$ respectively. After the ring closure, ring carbons (5'-C and $\left.4^{\prime}-\mathrm{C}\right)$ of compound $9 \mathrm{c}$ gave signals at 62.88 and 40.85 ppm, respectively. In the ESI-MS spectra, molecular ion $[\mathrm{M}]^{+}$peaks, which were the base peaks, provided the molecular formula of all synthesized compounds $\mathbf{8 a - 8 h}$ and $9 \mathbf{a}-9 \mathbf{h}$. Microanalysis results are within $\pm 0.4 \%$ of the theoretical values.

Antimicrobial activity studies: Antifungal and antibacterial activities of the 2-thioxoquinazolinone (5), 2-hydrazinoquinazolinone (6), 2-( $\mathrm{N}^{\prime}$-allylidene-hydrazino)quinazolinone (8) and 2-(4,5-dihydropyrazolyl)quinazolinone (9) compounds were tested by microdilution broth method as MIC values. The MIC values of the compounds are presented in the Table- 1 . These results indicate that compounds $\mathbf{8 a}$ through $\mathbf{8 h}$ showed the best antibacterial activity against gram positive bacteria, especially E. faecalis. However compounds $\mathbf{8 d}$ and $\mathbf{8 h}$ had no significant activity against $S$. aureus. All compounds showed similar antifungal activity. Their antifungal activity was not

TABLE-1

ANTIFUNGAL AND ANTIBACTERIAL ACTIVITIES OF 2-THIOXOQUINAZOLINONE (5), 2-HYDRAZINOQUINAZOLINONE (6), 2-(N'ALLYLIDENE-HYDRAZINO)-QUINAZOLINONES (8) AND 2-(4,5-DIHYDROPYRAZOLYL)-QUINAZOLINONES (9) (MIC in $\mu \mathrm{g} / \mathrm{mL})$

\begin{tabular}{|c|c|c|c|c|c|c|c|}
\hline Compound No. & $\begin{array}{c}\text { S. aureus } \\
\text { ATCC } 29213\end{array}$ & $\begin{array}{c}\text { E. faecalis } \\
\text { ATCC } 29212\end{array}$ & $\begin{array}{c}\text { E. coli } \\
\text { ATCC } 25922\end{array}$ & $\begin{array}{c}P . \text { aeruginosa } \\
\text { ATCC } 27853 \\
\end{array}$ & $\begin{array}{c}\text { C. albicans } \\
\text { ATCC } 90028\end{array}$ & $\begin{array}{c}\text { C. krusei } \\
\text { ATCC } 6258\end{array}$ & $\begin{array}{c}\text { C. parapsilosis } \\
\text { ATCC } 90018\end{array}$ \\
\hline 5 & 256 & 256 & 256 & 256 & 32 & 128 & 128 \\
\hline 6 & 64 & 128 & 256 & 256 & ND & 64 & 128 \\
\hline $8 \mathrm{a}$ & 128 & 32 & 256 & 512 & ND & 128 & 128 \\
\hline $8 \mathrm{~b}$ & 128 & 16 & 256 & 256 & ND & 128 & 128 \\
\hline $8 c$ & 128 & 16 & 256 & 256 & 64 & 128 & 128 \\
\hline $8 d$ & $>1024$ & 64 & 1024 & 1024 & 256 & 256 & 128 \\
\hline $8 \mathrm{e}$ & 128 & 256 & 512 & 256 & 128 & 128 & 128 \\
\hline $8 f$ & 64 & 32 & 256 & 256 & 128 & 128 & 256 \\
\hline $8 g$ & 256 & 32 & 256 & 256 & 128 & 128 & 256 \\
\hline $8 \mathrm{~h}$ & 1024 & 64 & 256 & 512 & 128 & 128 & 128 \\
\hline $9 \mathrm{c}$ & 512 & 512 & 256 & 512 & 128 & 128 & 128 \\
\hline $9 d$ & 512 & $>1024$ & 512 & $>1024$ & 1024 & 128 & 128 \\
\hline $9 \mathrm{e}$ & 512 & $>1024$ & 1024 & 1024 & 64 & 128 & 128 \\
\hline $9 \mathrm{~h}$ & 1024 & 128 & 512 & 256 & 32 & 256 & 64 \\
\hline Fluconazole & - & - & - & - & 0.25 & 16 & 1 \\
\hline
\end{tabular}


promising, but compound $\mathbf{9 h}$ seem to possess activity with the higher MIC values, except for $C$. krusei strain.

A new series of 2-[N'-(1,3-diphenyl-allylidene)hydrazino]-3-ethyl-3H-quinazolin-4-one (8) and 2-(3,5diphenyl-4,5-dihydro-pyrazol-1-yl)-3-ethyl-3H-quinazolin-4one (9) compounds have been synthesized and screened for their antibacterial [Staphylococcus aureus (ATCC 29213), Enterococcus faecalis (ATCC 29212), Escherichia coli (ATCC 25922) and Pseudomonas aeruginosa (ATCC 27853)] and antifungal [Candida albicans (ATCC 90028), Candida parapsilosis (ATCC 90018) and C. krusei (ATCC 6258)] activities. 8 compounds were found most effective on bacteria E. faecalis with the higher MIC values whereas 9 h was most effective on C. albicans and C. parapsilosis fungies. With these results in our hand, future work is aimed to be done for the synthesis and activity studies of new quinazolinone compounds.

3-Ethyl-2-[N'-(3-phenyl-1-p-tolyl-allylidene)hydrazino]-3H-quinazolin-4-one (8a): Obtained according to the general procedure, by using $7 \mathbf{a}(222 \mathrm{mg}, 1.0 \mathrm{mmol})$, as a white solid (335 mg, $82 \%$ ); m.p. $163-164{ }^{\circ} \mathrm{C} ; \mathrm{R}_{\mathrm{f}} 0.35\left(\mathrm{CHCl}_{3}\right) ;{ }^{1} \mathrm{H}$ NMR $\left(400 \mathrm{MHz}, \mathrm{CDCl}_{3}\right) \delta_{H} 9.40(1 \mathrm{H}, \mathrm{br}-\mathrm{s}, \mathrm{NH}), 8.10(1 \mathrm{H}, \mathrm{d}$, $J=8.4 \mathrm{~Hz}, 5-\mathrm{CH}), 8.00\left(1 \mathrm{H}, \mathrm{d}, J=16.8 \mathrm{~Hz}, 3^{\prime}-\mathrm{CH}\right), 7.51(2 \mathrm{H}$, $\mathrm{d}, J=8.0 \mathrm{~Hz}, 2 \times \mathrm{CH}(\mathrm{Ar})), 7.48(1 \mathrm{H}, \mathrm{t}, J=7.6 \mathrm{~Hz}, 7-\mathrm{CH})$, $7.30(7 \mathrm{H}, \mathrm{m}, 7 \times \mathrm{CH}(\mathrm{Ar})), 7.09(1 \mathrm{H}, \mathrm{t}, J=7.6 \mathrm{~Hz}, 6-\mathrm{CH})$, $6.93(1 \mathrm{H}, \mathrm{d}, J=8.0 \mathrm{~Hz}, 8-\mathrm{CH}), 6.86\left(1 \mathrm{H}, \mathrm{d}, J=16.4,2^{\prime}-\mathrm{CH}\right)$, $4.37\left(2 \mathrm{H}, \mathrm{q}, J=7.0 \mathrm{~Hz}, \mathrm{NCH}_{2} \mathrm{CH}_{3}\right), 2.50\left(3 \mathrm{H}, \mathrm{s}, \mathrm{PhCH}_{3}\right), 1.48$ $\left(3 \mathrm{H}, \mathrm{t}, J=7.0 \mathrm{~Hz}, \mathrm{NCH}_{2} \mathrm{CH}_{3}\right) ;{ }^{13} \mathrm{C}-\mathrm{NMR}\left(100 \mathrm{MHz}, \mathrm{CDCl}_{3}\right)$ $\delta_{C} 160.85$ (4-C), $160.17\left(1^{\prime}-C\right), 148.94$ (2-C), 138.68 (2'-C), $136.91,135.26,134.70,129.50,129.34,128.99,128.91$, $128.82,128.39,127.38,127.16,122.20(\mathrm{Ar}-\mathrm{C}), 120.81$ (3'- $^{\prime}$ C), $36.59\left(\mathrm{NCH}_{2} \mathrm{CH}_{3}\right), 21.41\left(\mathrm{PhCH}_{3}\right), 12.59\left(\mathrm{NCH}_{2} \mathrm{CH}_{3}\right)$; $\mathrm{MS}$ (EI) $\mathrm{m} / \mathrm{z} 408.2\left(\mathrm{M}^{+}, 100 \%\right)$; Anal. calcd. for $\mathrm{C}_{26} \mathrm{H}_{24} \mathrm{~N}_{4} \mathrm{O}$ : C, 76.45; H, 5.92; N, 13.72. Found: C, 76.74; H, 6.28; N, $13.97 \%$.

2-\{N'-[3-(4-Chlorophenyl)-1-p-tolyl-allylidene]hydrazino\}-3-ethyl-3H-quinazolin-4-one (8b): Obtained according to the general procedure, by using $7 \mathbf{b}(256 \mathrm{mg}, 1.0 \mathrm{mmol})$, as a white solid (349 g, $79 \%)$; m.p. $178-179{ }^{\circ} \mathrm{C} ; \mathrm{R}_{\mathrm{f}} 0.38\left(\mathrm{CHCl}_{3}\right)$; ${ }^{1} \mathrm{H}$ NMR $\left(400 \mathrm{MHz}, \mathrm{CDCl}_{3}\right) \delta_{H} 9.44(1 \mathrm{H}, \mathrm{br}-\mathrm{s}, \mathrm{NH}), 8.15(1 \mathrm{H}$, $\mathrm{d}, J=8.0 \mathrm{~Hz}, 5-\mathrm{CH}), 8.05\left(1 \mathrm{H}, \mathrm{d}, J=16.6 \mathrm{~Hz}, 3^{\prime}-\mathrm{CH}\right), 7.56$ $(2 \mathrm{H}, \mathrm{d}, J=7.6 \mathrm{~Hz}, 2 \times \mathrm{CH}(\mathrm{Ar})), 7.55(1 \mathrm{H}, \mathrm{t}, J=7.7 \mathrm{~Hz}, 7-$ $\mathrm{CH}), 7.37(2 \mathrm{H}, \mathrm{d}, J=7.8 \mathrm{~Hz}, 2 \times \mathrm{CH}(\mathrm{Ar})), 7.32(2 \mathrm{H}, \mathrm{d}, J=8.1$ $\mathrm{Hz}, 2 \times \mathrm{CH}(\mathrm{Ar})), 7.23(2 \mathrm{H}, \mathrm{d}, J=8.0 \mathrm{~Hz}, 2 \times \mathrm{CH}(\mathrm{Ar})), 7.14$ $(1 \mathrm{H}, \mathrm{t}, J=7.6 \mathrm{~Hz}, 6-\mathrm{CH}), 7.03(1 \mathrm{H}, \mathrm{d}, J=8.1 \mathrm{~Hz}, 8-\mathrm{CH}), 6.90$ $\left(1 \mathrm{H}, \mathrm{d}, J=16.6,2^{\prime}-\mathrm{CH}\right), 4.36\left(2 \mathrm{H}, \mathrm{q}, \mathrm{J}=6.8 \mathrm{~Hz}, \mathrm{NCH}_{2} \mathrm{CH}_{3}\right)$, $2.42\left(3 \mathrm{H}, \mathrm{s}, \mathrm{PhCH}_{3}\right), 1.45\left(3 \mathrm{H}, \mathrm{t}, J=6.8 \mathrm{~Hz}, \mathrm{NCH}_{2} \mathrm{CH}_{3}\right) ;{ }^{13} \mathrm{C}$ NMR $\left(100 \mathrm{MHz}, \mathrm{CDCl}_{3}\right) \delta_{C} 160.51(4-C), 159.85\left(1^{\prime}-C\right)$, 148.98 (2-C), 138.57 (2'-C), 135.30, 134.94, 134.77, 132.90, $129.38,129.23,129.06,128.54,128.49,126.85,122.84$, 122.37 (Ar-C), 121.14 (3'-C), $36.75\left(\mathrm{NCH}_{2} \mathrm{CH}_{3}\right), 21.42$ $\left(\mathrm{PhCH}_{3}\right), 12.58\left(\mathrm{NCH}_{2} \mathrm{CH}_{3}\right)$; MS (EI) $m / z 442.1\left(\mathrm{M}^{+}, 100 \%\right)$, 444.1; Anal. Calcd. for $\mathrm{C}_{26} \mathrm{H}_{23} \mathrm{~N}_{4} \mathrm{OCl}$ : C, 70.50; H, 5.23; N, 12.65. Found: C, 70.85; H, 5.49; N, $12.93 \%$.

2-[N'-(1,3-Di-p-tolylallylidene)hydrazino]-3-ethyl-3Hquinazolin-4-one (8c): Obtained according to the general procedure, by using 7c $(236 \mathrm{~g}, 1.0 \mathrm{mmol})$, as a white solid (380 mg, $90 \%$ ); m.p. $172-173{ }^{\circ} \mathrm{C} ; \mathrm{R}_{\mathrm{f}} 0.40\left(\mathrm{CHCl}_{3}\right) ;{ }^{1} \mathrm{H} \mathrm{NMR}$ $\left(400 \mathrm{MHz}, \mathrm{CDCl}_{3}\right) \delta_{\mathrm{H}} 9.43(1 \mathrm{H}$, br-s, NH), $8.13(1 \mathrm{H}, \mathrm{d}, J=$
$7.9 \mathrm{~Hz}, 5-\mathrm{CH}), 8.06\left(1 \mathrm{H}, \mathrm{d}, J=16.4 \mathrm{~Hz}, 3^{\prime}-\mathrm{CH}\right), 7.58(2 \mathrm{H}, \mathrm{d}$, $J=8.0 \mathrm{~Hz}, 2 \times \mathrm{CH}(\mathrm{Ar})), 7.53(1 \mathrm{H}, \mathrm{t}, J=7.0 \mathrm{~Hz}, 7-\mathrm{CH}), 7.44$ $(2 \mathrm{H}, \mathrm{d}, J=8.0 \mathrm{~Hz}, 2 \times \mathrm{CH}(\mathrm{Ar})), 7.32(2 \mathrm{H}, \mathrm{d}, J=7.8 \mathrm{~Hz}, 2 \times$ $\mathrm{CH}(\mathrm{Ar})), 7.22(2 \mathrm{H}, \mathrm{d}, J=7.9 \mathrm{~Hz}, 2 \times \mathrm{CH}(\mathrm{Ar})), 7.13(1 \mathrm{H}, \mathrm{t}, J$ $=7.4 \mathrm{~Hz}, 6-\mathrm{CH}), 7.00(1 \mathrm{H}, \mathrm{d}, J=8.1 \mathrm{~Hz}, 8-\mathrm{CH}), 6.93(1 \mathrm{H}, \mathrm{d}$, $\left.J=16.6,2^{\prime}-\mathrm{CH}\right), 4.38\left(2 \mathrm{H}, \mathrm{q}, J=7.0 \mathrm{~Hz}, \mathrm{NCH}_{2} \mathrm{CH}_{3}\right), 2.49$ $\left(3 \mathrm{H}, \mathrm{s}, \mathrm{PhCH}_{3}\right), 2.41\left(3 \mathrm{H}, \mathrm{s}, \mathrm{PhCH}_{3}\right), 1.47(3 \mathrm{H}, \mathrm{t}, J=7.0 \mathrm{~Hz}$, $\left.\mathrm{NCH}_{2} \mathrm{CH} 3\right) ;{ }^{13} \mathrm{C} \mathrm{NMR}\left(100 \mathrm{MHz}, \mathrm{CDCl}_{3}\right) \delta_{C} 160.84(4-\mathrm{C})$, $160.44\left(1^{\prime}-C\right), 148.80(2-C), 138.69\left(2^{\prime}-C\right), 135.22,134.68$, $134.12,129.56,129.38,128.96,128.38,127.36,122.17$ (ArC), 119.84 (3'-C), $36.57\left(\mathrm{NCH}_{2} \mathrm{CH}_{3}\right), 21.40\left(\mathrm{PhCH}_{3}\right), 12.58$ $\left(\mathrm{NCH}_{2} \mathrm{CH}_{3}\right)$; MS (EI) $m / z, 422.2\left(\mathrm{M}^{+}, 100 \%\right)$; Anal. Calcd. for $\mathrm{C}_{27} \mathrm{H}_{26} \mathrm{~N}_{4} \mathrm{O}: \mathrm{C}, 76.75 ; \mathrm{H}, 6.20 ; \mathrm{N}, 13.26$. Found: C, 76.98; H, $6.51 ; \mathrm{N}, 13.57 \%$.

2-\{N'-[3-(4-Bromophenyl)-1-p-tolyl-allylidene]hydrazino\}-3-ethyl-3H-quinazolin-4-one (8d): Obtained according to the general procedure, by using $\mathbf{7 d}(301 \mathrm{mg}, 1.0 \mathrm{mmol})$, as a white solid (418 g, $86 \%$ ); m.p. $185-186{ }^{\circ} \mathrm{C} ; \mathrm{R}_{\mathrm{f}} 0.36\left(\mathrm{CHCl}_{3}\right)$; ${ }^{1} \mathrm{H} \mathrm{NMR}\left(400 \mathrm{MHz}, \mathrm{CDCl}_{3}\right) \delta_{H} 9.26(1 \mathrm{H}, \mathrm{br}-\mathrm{s}, \mathrm{NH}), 8.07(1 \mathrm{H}$, d, $J=7.6 \mathrm{~Hz}, 5-\mathrm{CH}), 8.00\left(1 \mathrm{H}, \mathrm{d}, J=16.7 \mathrm{~Hz}, 3^{\prime}-\mathrm{CH}\right), 7.50$ $(1 \mathrm{H}, \mathrm{t}, J=7.2 \mathrm{~Hz}, 7-\mathrm{CH}), 7.47(4 \mathrm{H}, \mathrm{d}, J=8.3 \mathrm{~Hz}, 4 \times \mathrm{CH}(\mathrm{Ar}))$, $7.33(2 \mathrm{H}, \mathrm{d}, J=8.4 \mathrm{~Hz}, 2 \times \mathrm{CH}(\mathrm{Ar})), 7.24(2 \mathrm{H}, \mathrm{d}, J=8.0 \mathrm{~Hz}$, $2 \times \mathrm{CH}(\mathrm{Ar})), 7.08(1 \mathrm{H}, \mathrm{t}, J=7.6 \mathrm{~Hz}, 6-\mathrm{CH}), 6.93(1 \mathrm{H}, \mathrm{d}, J=$ $8.1 \mathrm{~Hz}, 8-\mathrm{CH}), 6.78\left(1 \mathrm{H}, \mathrm{d}, J=16.7,2^{\prime}-\mathrm{CH}\right), 4.31(2 \mathrm{H}, \mathrm{q}, J=$ $\left.7.0 \mathrm{~Hz}, \mathrm{NCH}_{2} \mathrm{CH}_{3}\right), 2.44\left(3 \mathrm{H}, \mathrm{s}, \mathrm{PhCH}_{3}\right), 1.41(3 \mathrm{H}, \mathrm{t}, J=7.0$ $\left.\mathrm{Hz}, \mathrm{NCH}_{2} \mathrm{CH}_{3}\right) ;{ }^{13} \mathrm{C} \mathrm{NMR}\left(100 \mathrm{MHz}, \mathrm{CDCl}_{3}\right) \delta_{C} 160.42$ (4C), 159.37 (1'-C), 149.11 (2-C), 138.69 (2'-C), 136.78, 135.90, 134.48, 132.15, 131.95, 129.67, 129.29, 129.22, 128.93, 128.65, 128.57, 122.77 ( $\mathrm{Ar}-\mathrm{C}), 122.14\left(3^{\prime}-\mathrm{C}\right), 36.44\left(\mathrm{NCH}_{2} \mathrm{CH}_{3}\right), 21.42$ $\left(\mathrm{PhCH}_{3}\right), 12.64\left(\mathrm{NCH}_{2} \mathrm{CH}_{3}\right)$; MS (EI) $m / z 486.1\left(\mathrm{M}^{+}, 100 \%\right)$, 488.1; Anal. Calcd. for $\mathrm{C}_{26} \mathrm{H}_{23} \mathrm{~N}_{4} \mathrm{OBr}$ : C, 64.07; H, 4.76; N, 11,50. Found: C, 64.39; H, 5.12; N, $11.83 \%$.

2-\{N'-[1-(4-Chlorophenyl)-3-phenyl-allylidene]hydrazino -3-ethyl-3H-quinazolin-4-one (8e): Obtained according to the general procedure, by using 7 e (242 mg, 1.0 mmol), as a white solid (325 g, $76 \%$ ); m.p. 191-192 ${ }^{\circ} \mathrm{C} ; \mathrm{R}_{\mathrm{f}}$ $0.38\left(\mathrm{CHCl}_{3}\right) ;{ }^{1} \mathrm{H}$ NMR $\left(400 \mathrm{MHz}, \mathrm{CDCl}_{3}\right) \delta_{H} 9.43(1 \mathrm{H}, \mathrm{br}-\mathrm{s}$, $\mathrm{NH}), 8.14(1 \mathrm{H}, \mathrm{d}, J=7.9 \mathrm{~Hz}, 5-\mathrm{CH}), 8.10(1 \mathrm{H}, \mathrm{d}, J=16.7 \mathrm{~Hz}$, $\left.3^{\prime}-\mathrm{CH}\right), 7.62(2 \mathrm{H}, \mathrm{d}, J=8.5 \mathrm{~Hz}, 2 \times \mathrm{CH}(\mathrm{Ar})), 7.54(3 \mathrm{H}, \mathrm{m}, 7-$ $\mathrm{CH}$ and $2 \times \mathrm{CH}(\mathrm{Ar})), 7.48(2 \mathrm{H}, \mathrm{d}, J=8.5 \mathrm{~Hz}, 2 \times \mathrm{CH}(\mathrm{Ar}))$, $7.42(2 \mathrm{H}, \mathrm{t}, J=6.9 \mathrm{~Hz}, 2 \times \mathrm{CH}(\mathrm{Ar})), 7.38(1 \mathrm{H}, \mathrm{t}, J=7.0 \mathrm{~Hz}$, $2 \times \mathrm{CH}(\mathrm{Ar})), 7.16(1 \mathrm{H}, \mathrm{t}, J=8.0 \mathrm{~Hz}, 6-\mathrm{CH}), 7.04(1 \mathrm{H}, \mathrm{d}, J=$ $8.0 \mathrm{~Hz}, 8-\mathrm{CH}), 6.91\left(1 \mathrm{H}, \mathrm{d}, J=16.8,2^{\prime}-\mathrm{CH}\right), 4.37(2 \mathrm{H}, \mathrm{q}, J=$ $\left.7.0 \mathrm{~Hz}, \mathrm{NCH}_{2} \mathrm{CH}_{3}\right), 1.45\left(3 \mathrm{H}, \mathrm{t}, J=7.0 \mathrm{~Hz}, \mathrm{NCH}_{2} \mathrm{CH}_{3}\right) ;{ }^{13} \mathrm{C}$ NMR (100 MHz, $\left.\mathrm{CDCl}_{3}\right) \delta_{C} 160.13$ (4-C), 159.23 (1'-C), 149.35 (2-C), 138.83 (2'-C), 136.65, 135.27, 134.41, 130.60, $129.56,129.43,129.31,129.01,128.80,128.46,128.38$, 128.14, 127.92, 127.33, 122.13 (Ar-C), 120.42 (3'-C), 36.56 $\left(\mathrm{NCH}_{2} \mathrm{CH}_{3}\right), 12.45\left(\mathrm{NCH}_{2} \mathrm{CH}_{3}\right)$; MS (EI) $\mathrm{m} / z$ 428.1 $\left(\mathrm{M}^{+}\right.$, $100 \%$ ), 430.1; Anal. Calcd. for $\mathrm{C}_{25} \mathrm{H}_{21} \mathrm{~N}_{4} \mathrm{OCl}$ : C, 70.01; $\mathrm{H}$, 4.93; N, 13.06. Found: C, 70.29; H, 5.27; N, $13.23 \%$.

2-\{N'-[1,3-bis-(4-Chlorophenyl)allylidene]hydrazino\}3-ethyl-3H-quinazolin-4-one (8f): Obtained according to the general procedure, by using $7 \mathbf{f}(277 \mathrm{~m}, 1.0 \mathrm{mmol})$, as a white solid (374 mg, $81 \%$ ); m.p. 206-207 ${ }^{\circ} \mathrm{C} ; \mathrm{R}_{f} 0.36\left(\mathrm{CHCl}_{3}\right) ;{ }^{1} \mathrm{H}$ NMR $\left(400 \mathrm{MHz}, \mathrm{CDCl}_{3}\right) \delta_{H} 9.13(1 \mathrm{H}$, br-s, NH), $7.92(1 \mathrm{H}, \mathrm{d}$, $J=7.9 \mathrm{~Hz}, 5-\mathrm{CH}), 7.82\left(1 \mathrm{H}, \mathrm{d}, J=16.7 \mathrm{~Hz}, 3^{\prime}-\mathrm{CH}\right), 7.57(1 \mathrm{H}$, $\mathrm{t}, J=7.1 \mathrm{~Hz}, 7-\mathrm{CH}), 7.39(2 \mathrm{H}, \mathrm{d}, J=8.5 \mathrm{~Hz}, 2 \times \mathrm{CH}(\mathrm{Ar}))$, 
$7.27(2 \mathrm{H}, \mathrm{d}, J=8.5 \mathrm{~Hz}, 2 \times \mathrm{CH}(\mathrm{Ar})), 7.23(2 \mathrm{H}, \mathrm{d}, J=8.7 \mathrm{~Hz}$, $2 \times \mathrm{CH}(\mathrm{Ar})), 7.16(2 \mathrm{H}, \mathrm{d}, J=8.4 \mathrm{~Hz}, 2 \times \mathrm{CH}(\mathrm{Ar})), 6.95(1 \mathrm{H}$, $\mathrm{t}, J=7.2 \mathrm{~Hz}, 6-\mathrm{CH}), 6.81(1 \mathrm{H}, \mathrm{d}, J=8.2 \mathrm{~Hz}, 8-\mathrm{CH}), 6.63(1 \mathrm{H}$, $\left.\mathrm{d}, J=16.7,2^{\prime}-\mathrm{CH}\right), 4.15\left(2 \mathrm{H}, \mathrm{q}, J=7.0 \mathrm{~Hz}, \mathrm{NCH}_{2} \mathrm{CH}_{3}\right), 1.23$ $\left(3 \mathrm{H}, \mathrm{t}, J=7.0 \mathrm{~Hz}, \mathrm{NCH}_{2} \mathrm{CH}_{3}\right) ;{ }^{13} \mathrm{C} \mathrm{NMR}\left(100 \mathrm{MHz}, \mathrm{CDCl}_{3}\right)$ $\delta_{C} 160.36(4-C), 158.20\left(1^{\prime}-C\right), 149.45(2-C), 138.44\left(2^{\prime}-C\right)$, $136.78,135.18,134.80,130.53,129.84,129.54,129.29$, $129.08,128.94,128.60,128.48,128.38,128.00,127.93$, 122.34 (Ar-C), $120.99\left(3^{\prime}-C\right), 36.49\left(\mathrm{NCH}_{2} \mathrm{CH}_{3}\right), 12.63$ $\left(\mathrm{NCH}_{2} \mathrm{CH}_{3}\right)$; MS (EI) $\mathrm{m} / z, 462.1\left(\mathrm{M}^{+}, 100 \%\right), 464.1,466.1$; Anal. Calcd. for $\mathrm{C}_{25} \mathrm{H}_{20} \mathrm{~N}_{4} \mathrm{OCl}_{2}$ : C, 64.80; H, 4.35; N, 12.09 . Found: C, 65.16; H, 4.72; N, $12.30 \%$.

2-\{N'-[1,3-bis-(4-Chlorophenyl)allylidene]hydrazino\}3-ethyl-3H-quinazolin-4-one (8g): Obtained according to the general procedure, by using $7 \mathbf{g}(256 \mathrm{~g}, 1.0 \mathrm{mmol})$, as a white solid (324 mg, 74 \%); m.p. $182-183{ }^{\circ} \mathrm{C} ; \mathrm{R}_{f} 0.39\left(\mathrm{CHCl}_{3}\right) ;{ }^{1} \mathrm{H}$ NMR $\left(400 \mathrm{MHz}, \mathrm{CDCl}_{3}\right) \delta_{H} 9.48(1 \mathrm{H}$, br-s, NH), $8.13(1 \mathrm{H}, \mathrm{d}$, $J=7.9 \mathrm{~Hz}, 5-\mathrm{CH}), 7.83\left(1 \mathrm{H}, \mathrm{d}, J=16.2 \mathrm{~Hz}, 3^{\prime}-\mathrm{CH}\right), 7.55(1 \mathrm{H}$, $\mathrm{t}, J=8.0 \mathrm{~Hz}, 7-\mathrm{CH}), 7.57(2 \mathrm{H}, \mathrm{d}, J=8.1 \mathrm{~Hz}, 2 \times \mathrm{CH}(\mathrm{Ar}))$, $7.43(2 \mathrm{H}, \mathrm{d}, J=8.1 \mathrm{~Hz}, 2 \times \mathrm{CH}(\mathrm{Ar})), 7.26(2 \mathrm{H}, \mathrm{d}, J=8.0 \mathrm{~Hz}$, $2 \times \mathrm{CH}(\mathrm{Ar})), 7.22(2 \mathrm{H}, \mathrm{d}, J=7.9 \mathrm{~Hz}, 2 \times \mathrm{CH}(\mathrm{Ar})), 7.15(1 \mathrm{H}$, t, $J=8.0 \mathrm{~Hz}, 6-\mathrm{CH}), 7.05(1 \mathrm{H}, \mathrm{d}, J=8.2 \mathrm{~Hz}, 8-\mathrm{CH}), 6.89(1 \mathrm{H}$, $\left.\mathrm{d}, J=16.6,2^{\prime}-\mathrm{CH}\right), 4.37$ (2H, q, $\left.J=7.0 \mathrm{~Hz}, \mathrm{NCH}_{2} \mathrm{CH}_{3}\right), 2.43$ $\left(3 \mathrm{H}, \mathrm{s}, \mathrm{PhCH}_{3}\right), 1.45\left(3 \mathrm{H}, \mathrm{t}, J=7.0 \mathrm{~Hz}, \mathrm{NCH}_{2} \mathrm{CH}_{3}\right) ;{ }^{13} \mathrm{C} \mathrm{NMR}$ $\left(100 \mathrm{MHz}, \mathrm{CDCl}_{3}\right) \delta_{C} 160.41(4-C), 158.86\left(1^{\prime}-C\right), 149.15$ (2C), $138.96\left(2^{\prime}-C\right), 138.55,138.41,136.81,134.82,134.49$, $133.95,130.62,129.54,128.58,128.40,127.30,122.19$ (ArC), 119.59 (3'-C), $36.48\left(\mathrm{NCH}_{2} \mathrm{CH}_{3}\right), 21.63\left(\mathrm{PhCH}_{3}\right), 12.68$ $\left(\mathrm{NCH}_{2} \mathrm{CH}_{3}\right)$; MS (EI) $m / z$ $442.1\left(\mathrm{M}^{+}, 100 \%\right)$, 444.1; Anal. Calcd. for $\mathrm{C}_{26} \mathrm{H}_{23} \mathrm{~N}_{4} \mathrm{OCl}$ : C, 70,50; H, 5.23; N, 12.65. Found: C, 70.84; H, 5.57; N, $13.01 \%$.

2-\{ $\mathbf{N}^{\prime}$-[3-(4-Bromophenyl)-1-(4-chlorophenyl)allylidene]-hydrazino\}-3-ethyl-3H-quinazolin-4-one (8h): Obtained according to the general procedure, by using $\mathbf{7 h}$ (321 $\mathrm{mg}, 1.0 \mathrm{mmol})$, as a white solid (430 mg, $85 \%$ ); m.p. 218$219{ }^{\circ} \mathrm{C} ; \mathrm{R}_{f} 0.30\left(\mathrm{CHCl}_{3}\right) ;{ }^{1} \mathrm{H} \mathrm{NMR}\left(400 \mathrm{MHz}, \mathrm{CDCl}_{3}\right) \delta_{H} 9.24$ $(1 \mathrm{H}, \mathrm{br}-\mathrm{s}, \mathrm{NH}), 8.07(1 \mathrm{H}, \mathrm{d}, J=7.9 \mathrm{~Hz}, 5-\mathrm{CH}), 8.00(1 \mathrm{H}, \mathrm{d}, J$ $\left.=16.7 \mathrm{~Hz}, 3^{\prime}-\mathrm{CH}\right), 7.52(1 \mathrm{H}, \mathrm{t}, J=7.0 \mathrm{~Hz}, 7-\mathrm{CH}), 7.44(2 \mathrm{H}$, $\mathrm{d}, J=8.4 \mathrm{~Hz}, 2 \times \mathrm{CH}(\mathrm{Ar})), 7.41(2 \mathrm{H}, \mathrm{d}, J=8.4 \mathrm{~Hz}, 2 \times \mathrm{CH}(\mathrm{Ar}))$, $7.28(2 \mathrm{H}, \mathrm{d}, J=8.3 \mathrm{~Hz}, 2 \times \mathrm{CH}(\mathrm{Ar})), 7.26(2 \mathrm{H}, \mathrm{d}, J=8.4 \mathrm{~Hz}$, $2 \times \mathrm{CH}(\mathrm{Ar})), 7.10(1 \mathrm{H}, \mathrm{t}, J=7.6 \mathrm{~Hz}, 6-\mathrm{CH}), 7.02(1 \mathrm{H}, \mathrm{d}, J=$ $8.1 \mathrm{~Hz}, 8-\mathrm{CH}), 6.75\left(1 \mathrm{H}, \mathrm{d}, J=16.8,2^{\prime}-\mathrm{CH}\right), 4.32(2 \mathrm{H}, \mathrm{q}, J=$ $\left.7.0 \mathrm{~Hz}, \mathrm{NCH}_{2} \mathrm{CH}_{3}\right), 1.41\left(3 \mathrm{H}, \mathrm{t}, J=7.0 \mathrm{~Hz}, \mathrm{NCH}_{2} \mathrm{CH}_{3}\right) ;{ }^{13} \mathrm{C}$ NMR (100 MHz, $\left.\mathrm{CDCl}_{3}\right) \delta_{C} 160.21(4-C), 159.81\left(1^{\prime}-C\right)$, 149.03 (2-C), 138.42 (2'-C), 135.50, 135.24, 134.57, 134.11, $132.04,131.89,130.75,130.53,130.27,128.68,128.49$, 128.28, 127.94, 122.43 (Ar- $C$ ), $122.36\left(3^{\prime}-C\right), 36.32$ $\left(\mathrm{NCH}_{2} \mathrm{CH}_{3}\right), 12.47\left(\mathrm{NCH}_{2} \mathrm{CH}_{3}\right)$; MS (EI) $m / z, 506.0\left(\mathrm{M}^{+}, 100\right.$ \%), 508.0; Anal. Calcd. for $\mathrm{C}_{25} \mathrm{H}_{20} \mathrm{~N}_{4} \mathrm{OClBr}$ : C, 59.13; H, 3.97; N, 11.03. Found: C, 59.42; H, 4.35; N, $11.38 \%$.

3-Ethyl-2-(5-phenyl-3-p-tolyl-4,5-dihydropyrazol-1yl)-3H-quinazolin-4-one (9a): Obtained according to the general procedure, by using $\mathbf{8 a}(102 \mathrm{mg}, 0.5 \mathrm{mmol})$, as a white solid (173 g, $85 \%)$; m.p. $125-126{ }^{\circ} \mathrm{C} ; \mathrm{R}_{f} 0.39\left(\mathrm{CHCl}_{3}\right) ;{ }^{1} \mathrm{H}$ NMR $\left(400 \mathrm{MHz}, \mathrm{CDCl}_{3}\right) \delta_{H} 8.00(1 \mathrm{H}, \mathrm{d}, J=8.0 \mathrm{~Hz}, 5-\mathrm{CH})$, $7.51(2 \mathrm{H}, \mathrm{d}, J=8.0 \mathrm{~Hz}, 2 \times \mathrm{CH}(\mathrm{Ar})), 7.55(1 \mathrm{H}, \mathrm{t}, J=8.4 \mathrm{~Hz}$, 7-CH), $7.44(2 \mathrm{H}, \mathrm{d}, J=8.8 \mathrm{~Hz}, 2 \times \mathrm{CH}(\mathrm{Ar})), 7.39(2 \mathrm{H}, \mathrm{d}, J=$ $8.8 \mathrm{~Hz}, 2 \times \mathrm{CH}(\mathrm{Ar})), 7.35(1 \mathrm{H}, \mathrm{d}, J=8.0 \mathrm{~Hz}, 8-\mathrm{CH}), 7.25$ $(1 \mathrm{H}, \mathrm{t}, J=7.6 \mathrm{~Hz}, 6-\mathrm{CH}), 7.21(2 \mathrm{H}, \mathrm{d}, J=8.0 \mathrm{~Hz}, 2 \times \mathrm{CH}(\mathrm{Ar}))$, $5.94\left(1 \mathrm{H}, \mathrm{t}, J=11.2 \mathrm{~Hz}, 5^{\prime}-\mathrm{CH}\right), 4.40(2 \mathrm{H}, \mathrm{q}, J=7.0 \mathrm{~Hz}$, $\left.\mathrm{NCH}_{2} \mathrm{CH}_{3}\right), 3.75\left(1 \mathrm{H}, \mathrm{dd}, J=11.2\right.$ and $\left.17.0 \mathrm{~Hz}, 4^{\prime}-\mathrm{CH}_{\mathrm{a}}\right), 3.21$ $\left(1 \mathrm{H}, \mathrm{dd}, J=12.0\right.$ and $\left.16.9 \mathrm{~Hz}, 4^{\prime}-\mathrm{CH}_{\mathrm{b}}\right), 2.35\left(3 \mathrm{H}, \mathrm{s}, \mathrm{PhCH}_{3}\right)$, $1.48\left(3 \mathrm{H}, \mathrm{t}, J=7.0 \mathrm{~Hz}, \mathrm{NCH}_{2} \mathrm{CH}_{3}\right) ;{ }^{13} \mathrm{C} \mathrm{NMR}(100 \mathrm{MHz}$, $\left.\mathrm{CDCl}_{3}\right) \delta_{C} 162.48(4-C), 151.85(2-C), 149.42\left(3^{\prime}-C\right), 136.91$, $135.26,134.70,129.50,129.34,128.99,128.91,128.82$, 128.39, 127.38, 127.16, 122.20 (Ar-C), 63.57 (5'-C), 40.43 (4'-C), $36.65\left(\mathrm{NCH}_{2} \mathrm{CH}_{3}\right), 21.51\left(\mathrm{PhCH}_{3}\right), 13.73\left(\mathrm{NCH}_{2} \mathrm{CH}_{3}\right)$; MS (EI) $m / z$, $408.2\left(\mathrm{M}^{+}, 100 \%\right.$ ); Anal. Calcd. for $\mathrm{C}_{26} \mathrm{H}_{24} \mathrm{~N}_{4} \mathrm{O}$ : C, 76.45; H, 5.92; N, 13.72. Found: C, 76.79; H, 6.27; N, $14.05 \%$.

2-[5-(4-Chlorophenyl)-3-p-tolyl-4,5-dihydropyrazol-1yl]-3-ethyl-3H-quinazolin-4-one (9b): Obtained according to the general procedure, by using $\mathbf{8 b}(221 \mathrm{mg}, 0.5 \mathrm{mmol})$, as a white solid (181 g, $82 \%)$; m.p. $152-153{ }^{\circ} \mathrm{C} ; \mathrm{R}_{f} 0.42\left(\mathrm{CHCl}_{3}\right)$; ${ }^{1} \mathrm{H}$ NMR $\left(400 \mathrm{MHz}, \mathrm{CDCl}_{3}\right) \delta_{H} 8.12(1 \mathrm{H}, \mathrm{d}, J=8.1 \mathrm{~Hz}, 5-$ $\mathrm{CH}), 7.64(2 \mathrm{H}, \mathrm{d}, J=8.0 \mathrm{~Hz}, 2 \times \mathrm{CH}(\mathrm{Ar})), 7.59(1 \mathrm{H}, \mathrm{t}, J=7.6$ $\mathrm{Hz}, 7-\mathrm{CH}), 7.45(2 \mathrm{H}, \mathrm{d}, J=8.3 \mathrm{~Hz}, 2 \times \mathrm{CH}(\mathrm{Ar})), 7.39(2 \mathrm{H}, \mathrm{d}$, $J=7.8 \mathrm{~Hz}, 2 \times \mathrm{CH}(\mathrm{Ar})), 7.32(2 \mathrm{H}, \mathrm{d}, J=8.1 \mathrm{~Hz}, 8-\mathrm{CH}), 7.19$ $(1 \mathrm{H}, \mathrm{t}, J=7.6 \mathrm{~Hz}, 6-\mathrm{CH}), 7.07(2 \mathrm{H}, \mathrm{d}, J=8.1 \mathrm{~Hz}, 2 \times \mathrm{CH}(\mathrm{Ar}))$, $5.93\left(1 \mathrm{H}, \mathrm{t}, J=11.2 \mathrm{~Hz}, 5^{\prime}-\mathrm{CH}\right), 4.36(2 \mathrm{H}, \mathrm{q}, J=6.8 \mathrm{~Hz}$, $\left.\mathrm{NCH}_{2} \mathrm{CH}_{3}\right), 3.59\left(1 \mathrm{H}, \mathrm{dd}, J=11.2\right.$ and $\left.17.0 \mathrm{~Hz}, 4^{\prime}-\mathrm{CH}_{\mathrm{a}}\right), 3.29$ $\left(1 \mathrm{H}, \mathrm{dd}, J=12.0\right.$ and $\left.16.8 \mathrm{~Hz}, 4^{\prime}-\mathrm{CH}_{\mathrm{b}}\right), 2.48\left(3 \mathrm{H}, \mathrm{s}, \mathrm{PhCH}_{3}\right)$, $1.45\left(3 \mathrm{H}, \mathrm{t}, J=6.8 \mathrm{~Hz}, \mathrm{NCH}_{2} \mathrm{CH}_{3}\right) ;{ }^{13} \mathrm{C} \mathrm{NMR}(100 \mathrm{MHz}$, $\left.\mathrm{CDCl}_{3}\right) \delta_{C} 163.04(4-C), 152.43(2-C), 148.56\left(3^{\prime}-C\right), 136.30$, $135.94,135.77,131.90,128.38,128.23,128.06,127.54$, $127.49,125.85,121.84,121.37$ (Ar-C), $62.63\left(5^{\prime}-C\right), 40.71$ (4'-C), $36.23\left(\mathrm{NCH}_{2} \mathrm{CH}_{3}\right), 20.98\left(\mathrm{PhCH}_{3}\right), 13.08\left(\mathrm{NCH}_{2} \mathrm{CH}_{3}\right)$; MS (EI) $m / z 442.1\left(\mathrm{M}^{+}, 100 \%\right), 444.1$; Anal. Calcd. for $\mathrm{C}_{26} \mathrm{H}_{23} \mathrm{~N}_{4} \mathrm{OCl}$ : C, 70.50; H, 5.23; N, 12.65. Found: C, 70.83; H, 5.58; N, $12.93 \%$.

2-(3,5-Di-p-tolyl-4,5-dihydropyrazol-1-yl)-3-ethyl-3Hquinazolin-4-one (9c): Obtained according to the general procedure, by using $8 \mathbf{c}(211 \mathrm{mg}, 0.5 \mathrm{mmol})$, as a white solid (205 mg, $97 \%$ ); m.p. 136-137 ${ }^{\circ} \mathrm{C} ; \mathrm{R}_{f} 0.35\left(\mathrm{CHCl}_{3}\right) ;{ }^{1} \mathrm{H}$ NMR $\left(400 \mathrm{MHz}, \mathrm{CDCl}_{3}\right) \delta_{H} 8.12(1 \mathrm{H}, \mathrm{d}, J=7.9 \mathrm{~Hz}, 5-\mathrm{CH}), 7.58$ $(2 \mathrm{H}, \mathrm{d}, J=8.1 \mathrm{~Hz}, 2 \times \mathrm{CH}(\mathrm{Ar})), 7.54(1 \mathrm{H}, \mathrm{t}, J=8.0 \mathrm{~Hz}, 7-$ $\mathrm{CH}), 7.38(2 \mathrm{H}, \mathrm{d}, J=7.9 \mathrm{~Hz}, 2 \times \mathrm{CH}(\mathrm{Ar})), 7.37(1 \mathrm{H}, \mathrm{m}, 8-$ $\mathrm{CH}), 7.21(1 \mathrm{H}, \mathrm{m}, 6-\mathrm{CH}), 7.20(1 \mathrm{H}, \mathrm{d}, J=8.1 \mathrm{~Hz}, 2 \times \mathrm{CH}(\mathrm{Ar}))$, $7.10(1 \mathrm{H}, \mathrm{d}, J=7.9 \mathrm{~Hz}, 2 \times \mathrm{CH}(\mathrm{Ar})), 5.92(1 \mathrm{H}, \mathrm{t}, J=11.6 \mathrm{~Hz}$, $\left.5^{\prime}-\mathrm{CH}\right), 4.46\left(2 \mathrm{H}, \mathrm{q}, J=6.9 \mathrm{~Hz}, \mathrm{NCH}_{2} \mathrm{CH}_{3}\right), 3.68(1 \mathrm{H}, \mathrm{dd}, J=$ 11.2 and $\left.17.0 \mathrm{~Hz}, 4^{\prime}-\mathrm{CH}_{\mathrm{a}}\right), 3.19(1 \mathrm{H}, \mathrm{dd}, J=12.0$ and 17.0 $\left.\mathrm{Hz}, 4^{\prime}-\mathrm{CH}_{\mathrm{b}}\right), 2.40\left(3 \mathrm{H}, \mathrm{s}, \mathrm{PhCH}_{3}\right), 2.30\left(3 \mathrm{H}, \mathrm{s}, \mathrm{PhCH}_{3}\right), 1.54$ $\left(3 \mathrm{H}, \mathrm{t}, J=6.9 \mathrm{~Hz}, \mathrm{NCH}_{2} \mathrm{CH}_{3}\right) ;{ }^{13} \mathrm{C} \mathrm{NMR}\left(100 \mathrm{MHz}, \mathrm{CDCl}_{3}\right)$ $\delta_{C} 162.47(4-C), 151.81(2-C), 148.43\left(3^{\prime}-C\right), 136.20,132.65$, $129.59,128.53,128.35,128.27,126.52,126.08,125.47$, 125.05, 123.56, (Ar-C), $62.88\left(5^{\prime}-C\right), 40.85\left(4^{\prime}-C\right), 39.87$ $\left(\mathrm{NCH}_{2} \mathrm{CH}_{3}\right), 20.60\left(\mathrm{PhCH}_{3}\right), 20.23\left(\mathrm{PhCH}_{3}\right), 13.45$ $\left(\mathrm{NCH}_{2} \mathrm{CH}_{3}\right)$; MS (EI) $\mathrm{m} / \mathrm{z} 422.2\left(\mathrm{M}^{+}, 100 \%\right)$; Anal. Calcd. for $\mathrm{C}_{27} \mathrm{H}_{26} \mathrm{~N}_{4} \mathrm{O}$ : C, 76.75; H, 6.20; N, 13.26. Found: C, 77.06; H, $6.51 ; \mathrm{N}, 13.51 \%$.

2-[5-(4-Bromophenyl)-3-p-tolyl-4,5-dihydro-pyrazol1-yl]-3-ethyl-3H-quinazolin-4-one (9d): Obtained according to the general procedure, by using $\mathbf{8 d}(243 \mathrm{mg}, 0.5 \mathrm{mmol})$, as a white solid (231 g, $95 \%)$; m.p. $174-175{ }^{\circ} \mathrm{C} ; \mathrm{R}_{f} 0.42\left(\mathrm{CHCl}_{3}\right)$; ${ }^{1} \mathrm{H}$ NMR $\left(400 \mathrm{MHz}, \mathrm{CDCl}_{3}\right) \delta_{H} 8.00(1 \mathrm{H}, \mathrm{d}, J=8.0 \mathrm{~Hz}, 5-$ $\mathrm{CH}), 7.58(2 \mathrm{H}, \mathrm{d}, J=8.0 \mathrm{~Hz}, 2 \times \mathrm{CH}(\mathrm{Ar})), 7.55(1 \mathrm{H}, \mathrm{t}, J=8.4$ $\mathrm{Hz}, 7-\mathrm{CH}), 7.44(2 \mathrm{H}, \mathrm{d}, J=8.8 \mathrm{~Hz}, 2 \times \mathrm{CH}(\mathrm{Ar})), 7.39(2 \mathrm{H}, \mathrm{d}$, $J=8.8 \mathrm{~Hz}, 2 \times \mathrm{CH}(\mathrm{Ar})), 7.35(1 \mathrm{H}, \mathrm{d}, J=8.0 \mathrm{~Hz}, 8-\mathrm{CH}), 7.25$ 
$(1 \mathrm{H}, \mathrm{t}, J=7.6 \mathrm{~Hz}, 6-\mathrm{CH}), 7.21(2 \mathrm{H}, \mathrm{d}, J=8.0 \mathrm{~Hz}, 2 \times \mathrm{CH}(\mathrm{Ar}))$, $5.94\left(1 \mathrm{H}, \mathrm{t}, J=11.2 \mathrm{~Hz}, 5^{\prime}-\mathrm{CH}\right), 4.46(2 \mathrm{H}, \mathrm{q}, J=6.8 \mathrm{~Hz}$, $\left.\mathrm{NCH}_{2} \mathrm{CH}_{3}\right), 3.70\left(1 \mathrm{H}, \mathrm{dd}, J=11.2\right.$ and $\left.17.0 \mathrm{~Hz}, 4^{\prime}-\mathrm{CH}_{\mathrm{a}}\right), 3.16$ $\left(1 \mathrm{H}, \mathrm{dd}, J=12.0\right.$ and $\left.16.8 \mathrm{~Hz}, 4^{\prime}-\mathrm{CH}_{\mathrm{b}}\right), 2.41\left(3 \mathrm{H}, \mathrm{s}, \mathrm{PhCH}_{3}\right)$, $2.30\left(3 \mathrm{H}, \mathrm{s}, \mathrm{PhCH}_{3}\right), 1.55\left(3 \mathrm{H}, \mathrm{t}, J=7.2 \mathrm{~Hz}, \mathrm{NCH}_{2} \mathrm{CH}_{3}\right) ;{ }^{13} \mathrm{C}$ NMR (100 MHz, $\left.\mathrm{CDCl}_{3}\right) \delta_{C} 162.24(4-C), 151.73(2-C)$, $149.31\left(3^{\prime}-C\right), 135.46,133.90,132.01,131.80,129.50,129.43$, $129.30,129.18,129.12,128.96,128.50,126.98,126.41$, 125.79, 124.46, 122.36 (Ar- $C), 63.38\left(5^{\prime}-C\right), 40.35\left(4^{\prime}-C\right)$, $36.95\left(\mathrm{NCH}_{2} \mathrm{CH}_{3}\right), 21.42\left(\mathrm{PhCH}_{3}\right), 13.92\left(\mathrm{NCH}_{2} \mathrm{CH}_{3}\right) ; \mathrm{MS}(\mathrm{EI})$ $m / z 486.1\left(\mathrm{M}^{+}, 100 \%\right)$, 488.1; Anal. Calcd. for $\mathrm{C}_{26} \mathrm{H}_{23} \mathrm{~N}_{4} \mathrm{OBr}$ : C, 64.07; H, 4.76; N, 11.50. Found: C, 64.36; H, 4.97; N, $11.83 \%$.

2-[3-(4-Chlorophenyl)-5-phenyl-4,5-dihydro-pyrazol1-yl]-3-ethyl-3H-quinazolin-4-one (9e): Obtained according to the general procedure, by using $8 \mathbf{e}$ ( $214 \mathrm{mg}, 0.5 \mathrm{mmol})$, as a white solid (199 mg, $93 \%$ ); m.p. $165-166{ }^{\circ} \mathrm{C} ; \mathrm{R}_{f} 0.43$ $\left(\mathrm{CHCl}_{3}\right) ;{ }^{1} \mathrm{H}$ NMR $\left(400 \mathrm{MHz}, \mathrm{CDCl}_{3}\right) \delta_{H} 8.14(1 \mathrm{H}, \mathrm{d}, J=8.0$ $\mathrm{Hz}, 5-\mathrm{CH}), 7.63(2 \mathrm{H}, \mathrm{d}, J=8.8 \mathrm{~Hz}, 2 \times \mathrm{CH}(\mathrm{Ar})), 7.55(1 \mathrm{H}, \mathrm{t}$, $J=8.0 \mathrm{~Hz}, 7-\mathrm{CH}), 7.49(2 \mathrm{H}, \mathrm{d}, J=7.2 \mathrm{~Hz}, 2 \times \mathrm{CH}(\mathrm{Ar})), 7.40$ $(1 \mathrm{H}, \mathrm{m}, 8-\mathrm{CH}) 7.40(2 \mathrm{H}, \mathrm{d}, J=8.8 \mathrm{~Hz}, 2 \times \mathrm{CH}(\mathrm{Ar})), 7.32$ $(2 \mathrm{H}, \mathrm{t}, J=7.6 \mathrm{~Hz}, 2 \times \mathrm{CH}(\mathrm{Ar})), 7.25(1 \mathrm{H}, \mathrm{t}, J=8.0 \mathrm{~Hz}, 2 \times$ $\mathrm{CH}(\mathrm{Ar})), 7.25(1 \mathrm{H}, \mathrm{t}, J=8.0,6-\mathrm{CH}), 6.03(1 \mathrm{H}, \mathrm{t}, J=11.4 \mathrm{~Hz}$, $\left.5^{\prime}-\mathrm{CH}\right), 4.46\left(2 \mathrm{H}, \mathrm{q}, J=6.9 \mathrm{~Hz}, \mathrm{NCH}_{2} \mathrm{CH}_{3}\right), 3.71(1 \mathrm{H}, \mathrm{dd}, J=$ 11.3 and $\left.16.9 \mathrm{~Hz}, 4^{\prime}-\mathrm{CH}_{\mathrm{a}}\right), 3.21(1 \mathrm{H}, \mathrm{dd}, J=12.0$ and 16.9 $\left.\mathrm{Hz}, 4^{\prime}-\mathrm{CH}_{\mathrm{b}}\right), 1.54\left(3 \mathrm{H}, \mathrm{t}, J=6.9 \mathrm{~Hz}, \mathrm{NCH}_{2} \mathrm{CH}_{3}\right) ;{ }^{13} \mathrm{C} \mathrm{NMR}$ $\left(100 \mathrm{MHz}, \mathrm{CDCl}_{3}\right) \delta_{C} 162.89(4-C), 151.95(2-C), 148.96\left(3^{\prime}-\right.$ C), 136.12, 133.81, 130.49, 130.06, 129.65, 129.09, 128.83, $128.69,127.87,127.56,127.34,127.21,127.02,125.72$, 124.86 (Ar-C), $64.27\left(5^{\prime}-C\right), 41.67\left(4^{\prime}-C\right), 40.89\left(\mathrm{NCH}_{2} \mathrm{CH}_{3}\right)$, $14.37\left(\mathrm{NCH}_{2} \mathrm{CH}_{3}\right)$; $\mathrm{MS}(\mathrm{EI}) \mathrm{m} / z 428.1\left(\mathrm{M}^{+}, 100 \%\right), 430.1$; Anal. Calcd. for $\mathrm{C}_{25} \mathrm{H}_{21} \mathrm{~N}_{4} \mathrm{OCl}$ : C, 70.01; H, 4.93; N, 13.06 . Found: C, 70.29; H, 5.34; N, $13.32 \%$.

2-[3,5-bis-(4-Chlorophenyl)-4,5-dihydro-pyrazol-1-yl]3-ethyl-3H-quinazolin-4-one (9f): Obtained according to the general procedure, by using $\mathbf{8 f}(231 \mathrm{mg}, 0.5 \mathrm{mmol})$, as a white solid (199 mg, $86 \%$ ); m.p. $189-190{ }^{\circ} \mathrm{C} ; \mathrm{R}_{f} 0.41\left(\mathrm{CHCl}_{3}\right) ;{ }^{1} \mathrm{H}-$ NMR $\left(400 \mathrm{MHz}, \mathrm{CDCl}_{3}\right) \delta_{H} 8.25(1 \mathrm{H}, \mathrm{d}, J=8.0 \mathrm{~Hz}, 5-\mathrm{CH})$, $7.54(1 \mathrm{H}, \mathrm{t}, J=8.0 \mathrm{~Hz}, 7-\mathrm{CH}), 7.44(1 \mathrm{H}, \mathrm{m}, 8-\mathrm{CH}) 7.40(2 \mathrm{H}$, $\mathrm{d}, J=8.0 \mathrm{~Hz}, 2 \times \mathrm{CH}(\mathrm{Ar})), 7.35(2 \mathrm{H}, \mathrm{d}, J=8.0 \mathrm{~Hz}, 2 \times \mathrm{CH}(\mathrm{Ar}))$, $7.31(2 \mathrm{H}, \mathrm{d}, J=8.0 \mathrm{~Hz}, 2 \times \mathrm{CH}(\mathrm{Ar})), 7.28(2 \mathrm{H}, \mathrm{d}, J=8.0 \mathrm{~Hz}$, $2 \times \mathrm{CH}(\mathrm{Ar})), 7.20(1 \mathrm{H}, \mathrm{t}, J=8.0,6-\mathrm{CH}), 6.12(1 \mathrm{H}, \mathrm{t}, J=11.2$ $\left.\mathrm{Hz}, 5^{\prime}-\mathrm{CH}\right), 4.36\left(2 \mathrm{H}, \mathrm{q}, J=7.0 \mathrm{~Hz}, \mathrm{NCH}_{2} \mathrm{CH}_{3}\right), 3.78(1 \mathrm{H}, \mathrm{dd}$, $J=11.2$ and $\left.17.0 \mathrm{~Hz}, 4^{\prime}-\mathrm{CH}_{\mathrm{a}}\right), 3.25(1 \mathrm{H}, \mathrm{dd}, J=12.0$ and 17.0 $\left.\mathrm{Hz}, 4^{\prime}-\mathrm{CH}_{\mathrm{b}}\right), 1.63\left(3 \mathrm{H}, \mathrm{t}, J=7.0 \mathrm{~Hz}, \mathrm{NCH}_{2} \mathrm{CH}_{3}\right) .{ }^{13} \mathrm{C} \mathrm{NMR}$ $\left(100 \mathrm{MHz}, \mathrm{CDCl}_{3}\right) \delta_{C} 161.78(4-C), 152.43(2-C), 149.11\left(3^{\prime}-\right.$ C), 136.78, 135.18, 134.80, 130.53, 129.84, 129.54, 129.29, $129.08,128.94,128.60,128.48,128.38,128.00,127.93$, $122.34(\mathrm{Ar}-C), 64.36\left(5^{\prime}-C\right), 41.73\left(4^{\prime}-C\right), 40.76\left(\mathrm{NCH}_{2} \mathrm{CH}_{3}\right)$, $13.89\left(\mathrm{NCH}_{2} \mathrm{CH}_{3}\right)$; $\mathrm{MS}(\mathrm{EI}) \mathrm{m} / \mathrm{z} 462.1\left(\mathrm{M}^{+}, 100 \%\right), 464.1$, 466.1; Anal. Calcd. for $\mathrm{C}_{25} \mathrm{H}_{20} \mathrm{~N}_{4} \mathrm{OCl}_{2}$ : C, 64.80; H, 4.35; N, 12.09. Found: C, 65.16; H, 4.64; N, $12.33 \%$.

2-[3-(4-Chlorophenyl)-5-p-tolyl-4,5-dihydro-pyrazol1-yl]-3-ethyl-3H-quinazolin-4-one (9g): Obtained according to the general procedure, by using $8 \mathbf{g}(110 \mathrm{mg}, 0.5 \mathrm{mmol})$, as a white solid (183 mg, $83 \%$ ); m.p. $173-174{ }^{\circ} \mathrm{C} ; \mathrm{R}_{f} 0.44$ $\left(\mathrm{CHCl}_{3}\right) ;{ }^{1} \mathrm{H} \mathrm{NMR}\left(400 \mathrm{MHz}, \mathrm{CDCl}_{3}\right) \delta_{H} 8.14(1 \mathrm{H}, \mathrm{d}, J=7.8$ $\mathrm{Hz}, 5-\mathrm{CH}), 7.61(2 \mathrm{H}, \mathrm{d}, J=8.1 \mathrm{~Hz}, 2 \times \mathrm{CH}(\mathrm{Ar})), 7.61(1 \mathrm{H}, \mathrm{t}$, $J=7.6 \mathrm{~Hz}, 7-\mathrm{CH}), 7.38(2 \mathrm{H}, \mathrm{d}, J=8.0 \mathrm{~Hz}, 2 \times \mathrm{CH}(\mathrm{Ar})), 7.30$
$(1 \mathrm{H}, \mathrm{d}, J=8.0 \mathrm{~Hz}, 8-\mathrm{CH}), 7.22(1 \mathrm{H}, \mathrm{t}, J=7.4,6-\mathrm{CH}), 7.17$ $(1 \mathrm{H}, \mathrm{d}, J=8.1 \mathrm{~Hz}, 2 \times \mathrm{CH}(\mathrm{Ar})), 7.08(1 \mathrm{H}, \mathrm{d}, J=7.9 \mathrm{~Hz}, 2 \times$ $\mathrm{CH}(\mathrm{Ar})), 5.79\left(1 \mathrm{H}, \mathrm{t}, J=11.6 \mathrm{~Hz}, 5^{\prime}-\mathrm{CH}\right), 4.58(2 \mathrm{H}, \mathrm{q}, J=6.9$ $\left.\mathrm{Hz}, \mathrm{NCH}_{2} \mathrm{CH}_{3}\right), 3.74\left(1 \mathrm{H}, \mathrm{dd}, J=11.4\right.$ and $17.0 \mathrm{~Hz}, 4^{\prime}-\mathrm{CH}_{\mathrm{a}}$ ), $3.32\left(1 \mathrm{H}, \mathrm{dd}, J=12.0\right.$ and $\left.17.0 \mathrm{~Hz}, 4^{\prime}-\mathrm{CH}_{\mathrm{b}}\right), 2.37(3 \mathrm{H}, \mathrm{s}$, $\left.\mathrm{PhCH}_{3}\right), 1.63\left(3 \mathrm{H}, \mathrm{t}, J=6.9 \mathrm{~Hz}, \mathrm{NCH}_{2} \mathrm{CH}_{3}\right) ;{ }^{13} \mathrm{C} \mathrm{NMR}(100$ $\left.\mathrm{MHz}, \mathrm{CDCl}_{3}\right) \delta_{C} 162.37$ (4-C), $151.73(2-C), 148.28\left(3^{\prime}-C\right)$, $135.46,134.89,134.65,132.79,129.56,129.43,129.26$, 128.66, 128.43, 126.74, 122.65, $122.25(\mathrm{Ar}-\mathrm{C}), 62.66\left(5^{\prime}-C\right)$, $40.34\left(4^{\prime}-\mathrm{C}\right), 39.54\left(\mathrm{NCH}_{2} \mathrm{CH}_{3}\right), 20.53\left(\mathrm{PhCH}_{3}\right), 13.19$ $\left(\mathrm{NCH}_{2} \mathrm{CH}_{3}\right)$; MS (EI) $\mathrm{m} / 2442.1\left(\mathrm{M}^{+}, 100 \%\right)$, 444.1; Anal. Calcd. for $\mathrm{C}_{26} \mathrm{H}_{23} \mathrm{~N}_{4} \mathrm{OCl}$ : C, 70.50; H, 5.23; N, 12.65. Found: C, 70.82; H, 5.51; N, $12.88 \%$.

2-[5-(4-Bromophenyl)-3-(4-chloro-phenyl)-4,5-dihydropyrazol-1-yl]-3-ethyl-3H-quinazolin-4-one (9h): Obtained according to the general procedure, by using $\mathbf{8 h}(253 \mathrm{mg}, 0.5$ mmol), as a white solid (230 mg, $91 \%$ ); m.p. $210-211{ }^{\circ} \mathrm{C}$; $\mathrm{R}_{f}$ $0.36\left(\mathrm{CHCl}_{3}\right) ;{ }^{1} \mathrm{H} \mathrm{NMR}\left(400 \mathrm{MHz}, \mathrm{CDCl}_{3}\right) \delta_{H} 8.15(1 \mathrm{H}, \mathrm{d}, J=$ $7.6 \mathrm{~Hz}, 5-\mathrm{CH}), 7.63(2 \mathrm{H}, \mathrm{d}, J=8.8 \mathrm{~Hz}, 2 \times \mathrm{CH}(\mathrm{Ar})), 7.57$ $(1 \mathrm{H}, \mathrm{t}, J=8.0 \mathrm{~Hz}, 7-\mathrm{CH}), 7.45(2 \mathrm{H}, \mathrm{d}, J=8.4 \mathrm{~Hz}, 2 \times \mathrm{CH}(\mathrm{Ar}))$, $7.40(2 \mathrm{H}, \mathrm{d}, J=8.4 \mathrm{~Hz}, 2 \times \mathrm{CH}(\mathrm{Ar})), 7.39(2 \mathrm{H}, \mathrm{d}, J=8.4 \mathrm{~Hz}$, $2 \times \mathrm{CH}(\mathrm{Ar})), 7.35(1 \mathrm{H}, \mathrm{d}, J=8.0 \mathrm{~Hz}, 8-\mathrm{CH}), 7.27(1 \mathrm{H}, \mathrm{t}, J=$ 7.2, 6-CH), $5.95\left(1 \mathrm{H}, \mathrm{t}, J=12.0 \mathrm{~Hz}, 5^{\prime}-\mathrm{CH}\right), 4.44(2 \mathrm{H}, \mathrm{q}, J=$ $\left.7.2 \mathrm{~Hz}, \mathrm{NCH}_{2} \mathrm{CH}_{3}\right), 3.69\left(1 \mathrm{H}, \mathrm{dd}, J=11.2\right.$ and $16.8 \mathrm{~Hz}, 4^{\prime}-$ $\left.\mathrm{CH}_{\mathrm{a}}\right), 3.17\left(1 \mathrm{H}, \mathrm{dd}, J=12.0\right.$ and $\left.17.0 \mathrm{~Hz}, 4^{\prime}-\mathrm{CH}_{\mathrm{b}}\right), 1.52(3 \mathrm{H}$, $\left.\mathrm{t}, J=7.1 \mathrm{~Hz}, \mathrm{NCH}_{2} \mathrm{CH}_{3}\right) ;{ }^{13} \mathrm{C} \mathrm{NMR}\left(100 \mathrm{MHz}, \mathrm{CDCl}_{3}\right) \delta_{\mathrm{C}}$ 163.10 (4-C), 152.21 (2-C), 149.10 (3'-C), 136.34, 133.91, $131.96,131.73,129.19,129.00,127.39,126.95,124.82$, $122.03(\mathrm{Ar}-C), 63.91\left(5^{\prime}-C\right), 41.51\left(4^{\prime}-C\right), 40.58\left(\mathrm{NCH}_{2} \mathrm{CH}_{3}\right)$, $14.23\left(\mathrm{NCH}_{2} \mathrm{CH}_{3}\right)$; MS (EI) $\mathrm{m} / z 506.0\left(\mathrm{M}^{+}, 100 \%\right), 508.0$; Anal. Calcd. for $\mathrm{C}_{25} \mathrm{H}_{20} \mathrm{~N}_{4} \mathrm{OBrCl}$ : C, 59.13; H, 3.97; N, 11.03 . Found: C, 59.41; H, 4.39; N, $11.37 \%$.

\section{ACKNOWLEDGEMENTS}

The authors gratefully acknowledged the partial financial support from the Hacettepe University (BAB-03G046 and BAB-2009, 08 D05 301 002).

\section{REFERENCES}

1. R. Rajput and A.P. Mishra, Int. J. Pharm. Pharm. Sci., 4, 66 (2012).

2. A.S. El-Azab, Phosphorus, Sulfur, Silicon Rel. Elem., 182, 333 (2007).

3. N.A. Santagati, E. Bousquet, A. Spadaro and G. Ronsisvalle, IL Farmaco, 54, 780 (1999).

4. C. Parkanyi and D.S. Schmidt, J. Heterocycl. Chem., 37, 725 (2000).

5. I. Philipova, G. Dobrikov, K. Krumova and J. Kaneti, J. Heterocycl. Chem., 43, 1057 (2006).

6. M.B. Deshmukh, S.S. Patil, S.S. Patil and S.D. Jadhav, J. Heterocycl. Chem., 47, 1144 (2010).

7. M. Wang, Z. Song and T. Zhang, J. Heterocycl. Chem., 47, 468 (2010).

8. R.S. Giri, H.M. Thaker, T. Giordano, J. Williams, D. Rogers, V. Sudersanam and K.K. Vasu, Eur. J. Med. Chem., 44, 2184 (2009).

9. S.K. Kundu, M.P.D. Mahindaratne, M.V. Quintero, A. Bao and G.R. Negrete, ARKIVOC, 33 (2008).

10. V. Alagarsamy, S. Murugesan, K. Dhanabal, M. Murugan and E. De Clercq, Indian J. Pharm. Sci., 69, 304 (2007).

11. M.S. Mohamed, M.M. Kamel, E.M.M. Kassem, N. Abotaleb, S.I. Abd El-moez and M.F. Ahmed, Eur. J. Med. Chem., 45, 3311 (2010).

12. S.K. Kashaw, V. Kashaw, P. Mishra, N.K. Jain and J.P. Stables, Eur. J. Med. Chem., 44, 4335 (2009).

13. H. Georgey, N. Abdel-Gawad and S. Abbas, Molecules, 13, 2557 (2008).

14. S. Eguchi, T. Suzuki, T. Okawa, Y. Matsushita, E. Yashima and Y. Okamoto, J. Org. Chem. 61, 7316 (1996).

15. S. Hayao, H.J. Havera, W.G. Strycker, T.J. Leipzig, R.A. Kulp and H.E. Hartzler, J. Med.Chem., 8, 807 (1965). 
16. A. Rahman, A.A. Siddiqui, Int. J. Pharm. Sci. Drug Res., 2, 165 (2010).

17. M.A. Khalil, R. Soliman, A.M. Farghaly and A.A. Bekhit, Arch. Pharm., 327, 27 (1994).

18. A. Yesilada, S. Koyunoglu, N. Saygilia, E. Kupeli, E. Yesilada, E. Bedir and I. Khanc, Arch. Pharm. Pharm. Med. Chem., 337, 96 (2004).

19. W. Gaüss, H. J. Kabbe, W. Paülüs, H. J. Rosslenbroich and W. Brandes, US Patent 4,644,000 (1987).

20. A.A. El-Helby and M.H. Abdel Wahab, Egyptian J. Biomed. Sci., 11, 297 (2003).

21. Z. Li, H. Huang, H. Sun, H. Jiang and H. Liu, J. Comb. Chem., 10, 484 (2008).

22. G.N. Vassilev and N.G. Vassilev, Oxid. Commun., 30, 228 (2007).

23. I. Yavari and S. Beheshti, Helv. Chim. Acta, 94, 1825 (2011).

24. R. Murdoch, W. R. Tully and R. Westwood, J. Heterocycl. Chem., 23, 833 (1986).
25. K. Butler and M.W. Partridge, J. Chem. Soc. 1512 (1959).

26. K. Kottke, H. Kühmstedt and G. Griesner, Pharmazie, 38, 367 (1983).

27. W. Fathalla, M. Cajan and P. Pazdera, Molecules, 6, 557 (2001).

28. R. Jacquier and G. Maury, Bull. Soc. Chim. Fr., 306 (1967).

29. A. Hassner and M.J. Michelson, J. Org. Chem., 27, 298 (1962).

30. Clinical and Laboratory Standards Institute. Methods for Dilution Antimicrobial Susceptibility Tests for Bacteria that Grow Aerobically: Approved Standard, M07-A8, edn 8, Clinical and Laboratory Standards Institute, Wayne, PA (2008).

31. Clinical and Laboratory Standards Institute, Reference Method for Broth Dilution Antifungal Susceptibility Testing of Yeasts: Approved Standard, M27-A3, edn 3, Clinical and Laboratory Standards Institute, Wayne, PA (2008). 